\title{
Parkinsonism Secondary to Hydrocephalus Caused by Neurocysticercosis
}

Sebastião Carlos de Sousa Oliveira ${ }^{1}$, Dina Andressa Martins Monteiro ${ }^{2}$, Giselle Furtado Silva ${ }^{1}$, Lucas Tadeu Rocha Santos ${ }^{3}$, Espártaco Moraes Lima Ribeiro ${ }^{2}$

1. Neurological Surgery, Federal University of Ceara, Sobral, BRA 2. Neurology, Federal University of Ceara, Sobral, BRA 3. Internal Medicine, Federal University of Ceara, Sobral, BRA

Corresponding author: Sebastião Carlos de Sousa Oliveira, csosebastiao@gmail.com

\begin{abstract}
The diagnosis of parkinsonism is established by the presence of tremor, stiffness and bradykinesia alongside with neurological examination, requiring the exclusion of secondary causes such as stroke, hydrocephalus and infectious diseases. Included in this last category, neurocysticercosis is a disease caused by Taenia solium, with a variable clinical presentation that can include epileptic seizures, hydrocephalus and rarely parkinsonism. In the reported case, the syndrome is a consequence of lesions in the nigrostriatal dopaminergic pathway caused by the implant and mass effect of the cysticercus. The authors report a case of parkinsonism in a 59-year-old woman with a previous history of neurocysticercosis who presented with hydrocephalus on magnetic resonance imaging exam. The patient was treated with pharmacological therapy and ventriculoperitoneal shunt, progressing with amelioration of the symptoms presented.
\end{abstract}

Categories: Neurology, Infectious Disease, Neurosurgery

Keywords: neurocysticercosis, hydrocephalus, parkinsonism

\section{Introduction}

Parkinsonism is characterized by the triad of tremor, stiffness and bradykinesia [1]. Medical history and neurological examination are responsible for the diagnosis of parkinsonian syndromes, and tests such as MRI have the role of excluding underlying disease, such as stroke and normal pressure hydrocephalus. The differential diagnosis is much broader and includes neurodegenerative, metabolic, neoplastic, drug induced parkinsonism and infectious diseases, including neurocysticercosis (NC).

Cysticercosis is a disease caused by Taenia solium larva and has two syndromic presentations: extraneural and NC. The latter, in endemic countries, is considered the main cause of seizures in adults [2]. Other presentations of NC are focal neurological deficits, hydrocephalus, meningismus, arachnoiditis and some movement disorders, such as tremor, chorea, dystonia and parkinsonism.

Received 03/10/2020 Review began 04/08/2020 Review ended 04/21/2020 Published 04/29/2020

(c) Copyright 2020

Oliveira et al. This is an open access article distributed under the terms of the Creative Commons Attribution License CC-BY 4.0., which permits unrestricted use, distribution, and reproduction in any medium, provided the original author and source are credited.

\section{Case Presentation}

A 59-year-old woman was admitted with progressive symptoms of urinary incontinence, amnesia for recent events, failure to recognize family members and difficulty walking since four months ago. She had a widebased gait and stiffness predominating in the lower limbs, which later evolved to incapacity to walk and bradykinesia. The patient was diagnosed with NC 20 years ago and epilepsy approximately 15 years ago, treated with phenobarbital. She sought assistance from a neurologist, who performed a lumbar puncture in order to assess the persistence of symptoms after removing excess of cerebrospinal fluid (CSF). The lumbar puncture showed CSF of normal characteristics. The patient showed no improvement in symptoms after the procedure and was referred to the hospital for diagnostic investigation. Admission laboratory tests showed no pathological changes. Axial (Figure 1) and coronal (Figure 2) sections of a T2-weighted MRI of the brain revealed cystic lesions in the subcortical region with the presence of scolex associated with perilesional hyperintensity, suggestive of $\mathrm{NC}$ in vesicular stage associated with hydrocephalus and signals of transependymal resorption. 


\section{Cureus}

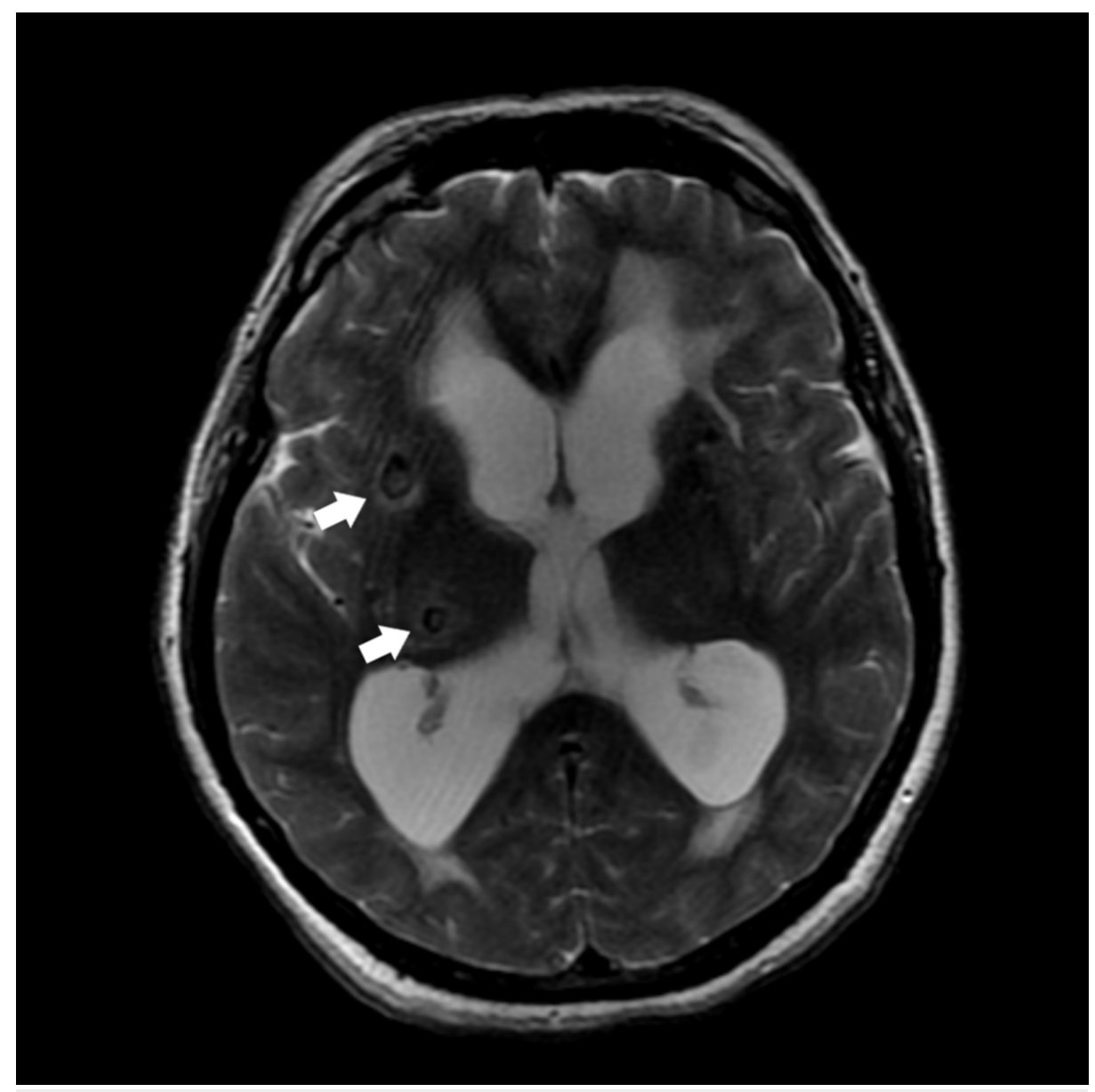

FIGURE 1: MRI T2-weighted axial.

T2-weighted axial MRI image showing subcortical cystic lesions with perilesional hyperintensity and presence of scolex (arrows). 


\section{Cureus}

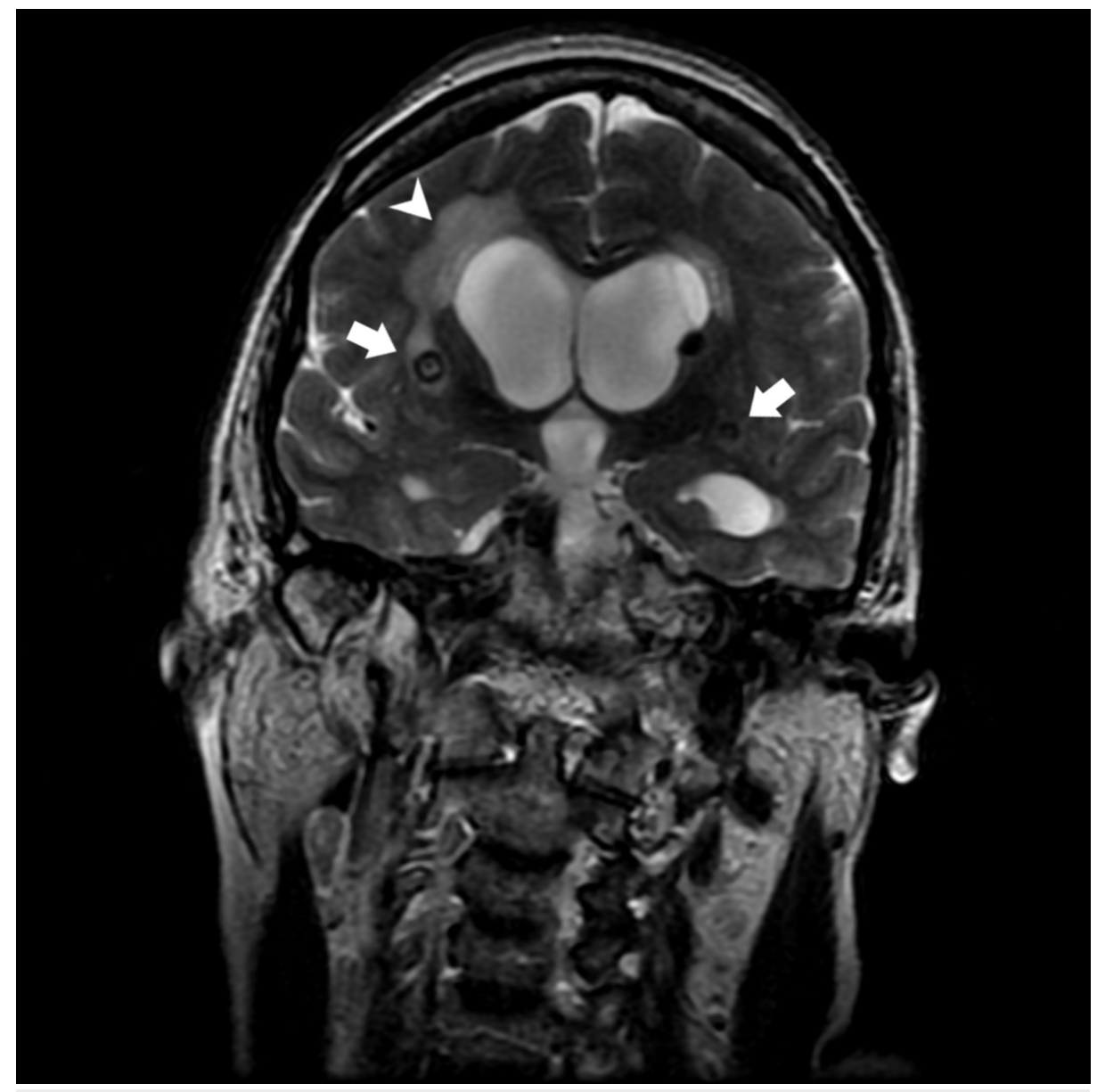

FIGURE 2: MRI T2-weighted coronal.

T2-weighted coronal MRI image showing cysticerci in brain parenchyma (left arrow) and subarachnoid space (right arrow) and signs of transependymal edema (arrowhead).

On T1-weighted sagittal section, the presence of a cysticercus in the foramen of Magendie was noted (Figure 3). The patient underwent ventriculoperitoneal shunt (VPS) insertion, and evolved with clinical improvement of the symptoms. A noncontrast-enhanced cranial CT (Figure 4) was performed to assess the postoperative status, which showed multiple cortical and subcortical calcifications and well-placed ventricular drain. The patient was discharged using albendazole and praziquantel for 14 days, on an outpatient basis. 


\section{Cureus}

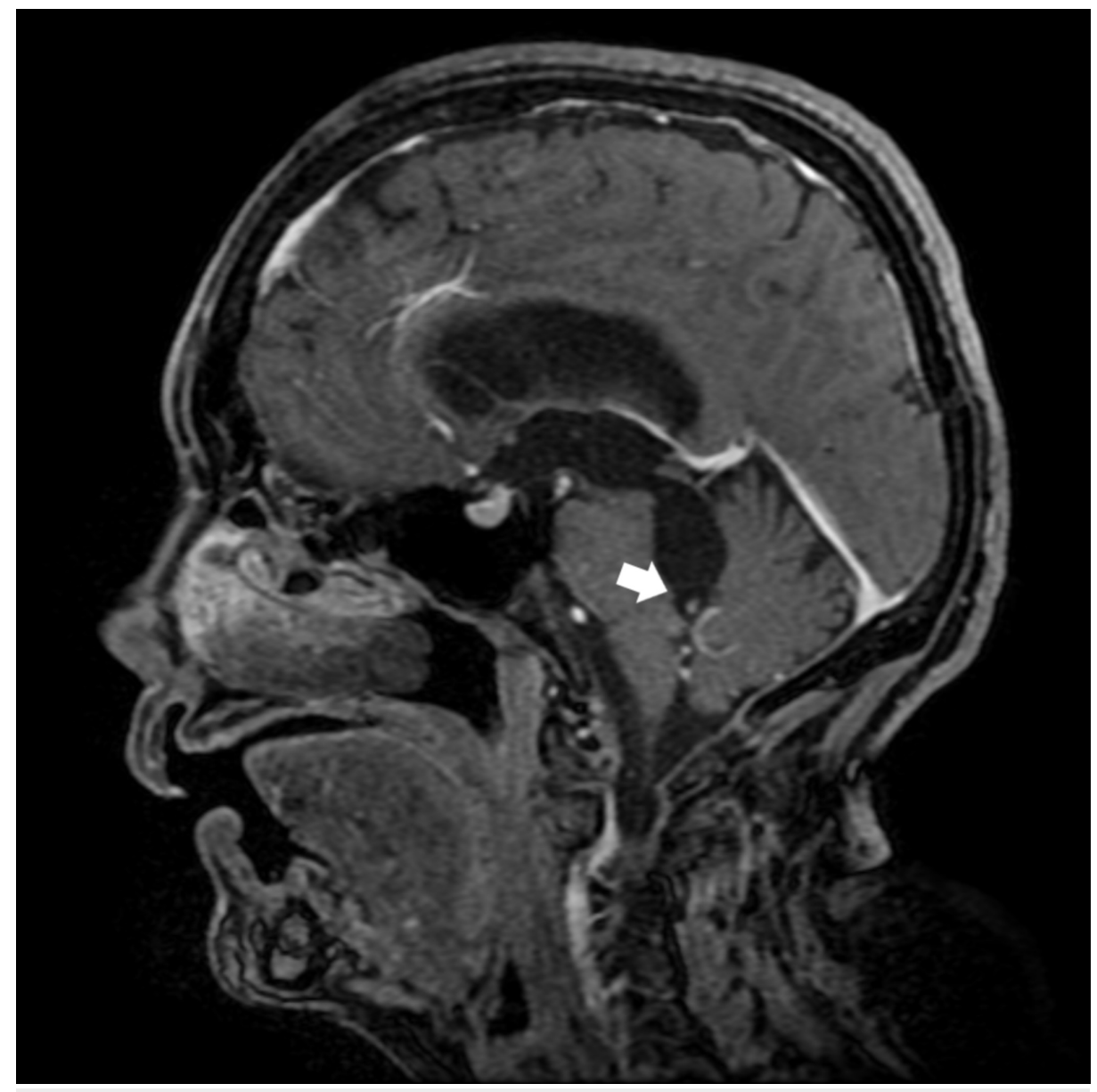

FIGURE 3: MRI T1-weighted sagittal.

T1-weighted sagittal MRI image showing the presence of cysticerci causing obstruction of the Magendie's foramen.

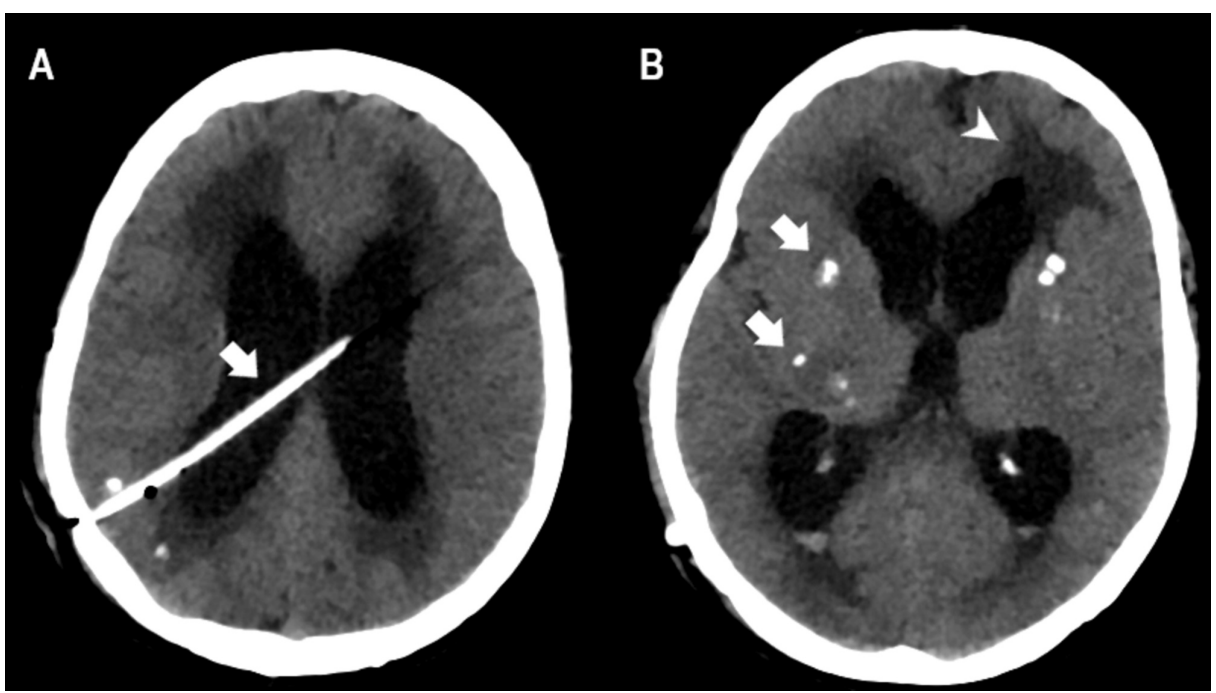

FIGURE 4: Noncontrast-enhanced cranial CT

(A) Noncontrast-enhanced cranial CT showing a well-placed ventricular drain (arrow). (B) Cranial CT showing multiple subcortical calcifications (arrows) associated with transependymal resorption (arrowhead). 


\section{Discussion}

$\mathrm{NC}$ is a parasitosis of the central nervous system caused by the helminth Taenia solium. This parasite has a life cycle in which man represents the only definitive host and the pig represents the intermediate host. Central nervous system infection in humans occurs when the individual accidentally ingests Taenia solium eggs, or when the larval stage has an affinity for the central nervous system tissue, causing NC [3]. NC can present several clinical manifestations according to the type, quantity and location of cysticerci in the nervous system. Common clinical manifestations of NC include epileptic seizures, cysticercotic meningitis, dementia, obstructive hydrocephalus and, more rarely, parkinsonism [3,4].

Parkinsonism, although rare, can be caused by lesions in the nigrostriatal dopaminergic pathway through tissue destruction. This destruction can be caused by direct implants of cysticerci in the tissue of the nigrostriatal pathway or by a mass effect resulting from the implantation of cysticerci in surrounding brain areas. The implantation of cysticerci in the IV ventricle or in the cisterna magna can prevent the flow of cerebrospinal fluid, resulting in hydrocephalus, which in turn can cause mass effect in the nigrostriatal pathway. Local edema resulting from the host's inflammatory reaction to implantation of cysticerci can also cause an increase in intracranial pressure and, therefore, generate or worsen lesions due to a mass effect in the nigrostriatal pathway [5].

The clinical presentation of NC is directly related to the location of the lesion. The disease in its intraparenchymal form, which accounts for $60 \%$ of the cases, is related to a convulsive condition due to the inflammatory response associated with degeneration or calcification of the cysticercus. Regarding the intraventricular form, which represents a total of $10 \%$ to $20 \%$ of the cases, it can cause obstructive hydrocephalus [2]. In the case described, both types of lesions are observed and may explain the parkinsonian syndrome. In a retrospective study, of the 590 patients studied, 23 (3.8\%) developed movement disorders, in which 15 (65\%) had parkinsonism [4]. Unlike what was observed in the case described, the time to onset of motor symptoms varied up to 140 days after diagnosis of NC. However, in other studies, this interval can vary up to 30 years, generally occurring between three and five years after infection [6]. In this group of patients, the clinical presentation of parkinsonism occurs differently, with bilateral involvement, axial hypokinesia, mild stiffness, infrequent resting tremor, instability, constant falls and frontal gait disorder. The exclusion of differential diagnoses is essential.

In the case presented, differential diagnoses were normal pressure hydrocephalus and Parkinson's disease. The first is marked by the triad of dementia, gait changes and urinary incontinence, being excluded as a diagnostic hypothesis due to the lack of improvement in symptoms after lumbar puncture, the subacute evolution and the presence of obstructive hydrocephalus [1]. Parkinson's disease was disregarded for the clinical case due to improvement of the symptoms with the treatment of the hydrocephalus and NC.

The diagnosis of NC can be made with the presence of one of the three absolute criteria, which are histopathological examination showing the parasite in the lesion, image examination showing scolex or direct visualization of the parasite in the fundus examination. In addition to the absolute criteria, there are two other categories of diagnostic criteria: neuroimaging criteria (Table 1) and clinical criteria (Table 2) [7].

Major neuroimaging criteria

Cystic lesions without a discernible scolex

Enhancing lesions

Multilobulated cystic lesions in the subarachnoid space
Confirmative neuroimaging criteria

Resolution of cystic lesions after cysticidal drug therapy

Spontaneous resolution of single small enhancing lesions

Migration of ventricular cysts documented on sequential neuroimaging studies

\section{Minor neuroimaging criteria}

Obstructive hydrocephalus (symmetric or asymmetric) or abnormal enhancement of basal leptomeninges

\section{TABLE 1: Neuroimaging criteria for the diagnosis of neurocysticercosis[7].}




\section{Cureus}

\section{Major clinical/exposure}

Detection of specific anticysticercal antibodies or cysticercal antigens by well-standardized immunodiagnostic tests

Cysticercosis outside the central nervous system
Minor clinical/exposure

Obstructive hydrocephalus (symmetric or asymmetric) or abnormal enhancement of basal leptomeninges

Evidence of a household contact with Taenia solium infection

TABLE 2: Clinical criteria for the diagnosis of neurocysticercosis [7].

The diagnosis of NC can be definitively established with the presence of two major neuroimaging criteria associated with a minor clinical criterion. The addition of a major neuroimaging criterion, a corroborative neuroimaging criterion and any clinical criterion also establishes the definitive diagnosis of the disease. In addition, the definitive diagnosis can also be established with the presence of a major neuroimaging criterion associated with two clinical criteria (at least one of them being a major criterion), having excluded other possible similar pathologies (Figure 5) [7].

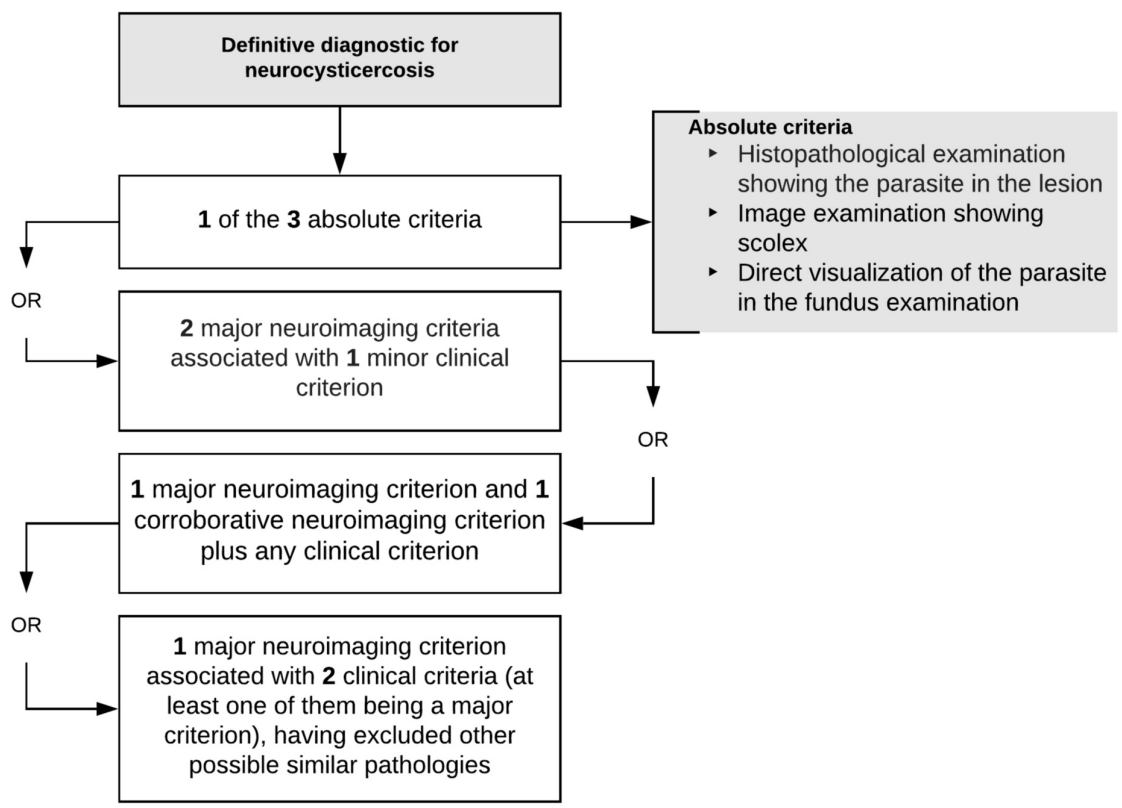

FIGURE 5: Definitive diagnostic for neurocysticercosis.

As for the clinical case presented in this article, the treatment for parkinsonian syndrome presented by the patient is mainly the treatment of the underlying etiology (NC and hydrocephalus), obtaining improvement with the insertion of a VPS. In cases of active cystic lesions, a recent study showed that the combined therapy of praziquantel $50 \mathrm{mg} / \mathrm{kg} /$ day and albendazole $15 \mathrm{mg} / \mathrm{kg} /$ day for 10 days was more effective than the use of a single drug, without causing greater side effects. At the beginning of treatment, corticosteroids are often used for a few days associated with cysticide therapy to minimize cerebral edema caused by the death of cysticerci. Dexamethasone and prednisone are the most frequently used medications [8].

The process of resolving acute infection can lead to the formation of granulomas, which can remain to cause the symptoms of the disease in the patient during this process. It is possible that calcified residues remain in the nervous system, which can generate clinical manifestations for a prolonged period of time. Therefore, the use of medications directed to these symptoms may be necessary. Due to the diversity of possible clinical manifestations of the disease, the treatment of $\mathrm{NC}$ is a very comprehensive topic, which can encompass several other specific therapeutic strategies, such as VPS and the use of levodopa [8].

\section{Conclusions}

$\mathrm{NC}$ is a disease that presents itself clinically in several ways, one of which is parkinsonism. Although rare, 
parkinsonism secondary to NC can cause numerous losses to the patient's life. Therefore, it is extremely relevant that the medical community knows how to recognize this condition, so that it is possible to establish an appropriate treatment for the patient and improve the individual's quality of life. However, it should be emphasized that additional investigation on the subject is needed in order to establish diagnoses and treatments with greater security.

\section{Additional Information \\ Disclosures}

Human subjects: Consent was obtained by all participants in this study. Conflicts of interest: In compliance with the ICMJE uniform disclosure form, all authors declare the following: Payment/services info: All authors have declared that no financial support was received from any organization for the submitted work. Financial relationships: All authors have declared that they have no financial relationships at present or within the previous three years with any organizations that might have an interest in the submitted work. Other relationships: All authors have declared that there are no other relationships or activities that could appear to have influenced the submitted work.

\section{References}

1. Postuma RB, Berg D, Stern M, et al.: MDS clinical diagnostic criteria for Parkinson's disease . Mov Disord Clin Pract. 2015, 30:1591-1601. 10.1002/mds.26424

2. White AC Jr, Coyle CM, Rajshekhar V, et al.: Diagnosis and treatment of neurocysticercosis: 2017 clinical practice guidelines by the Infectious Diseases Society of America (IDSA) and the American Society of Tropical Medicine and Hygiene (ASTMH). Clin Infect Dis. 2018, 22:e49-e75. 10.1093/cid/cix1084

3. Dermauw V, Van Den Broucke S, Van Bockstal L, et al.: Cysticercosis and taeniasis cases diagnosed at two referral medical institutions, Belgium, 1990 to 2015. Euro Surveill. 2019, 29:1-10. 10.2807/15607917.ES.2019.24.35.1800589

4. Alarcón F, Cedeño Y, de Yébenes JG: Parkinsonism and other movement disorders in 23 cases of neurocysticercosis. Clin Park Relat Disord. 2017, 42:47-53. 10.1016/j.parkreldis.2017.06.009

5. Prashantha DK, Netravathi M, Ravishankar S, et al.: Reversible parkinsonism following ventriculoperitoneal shunt in a patient with obstructive hydrocephalus secondary to intraventricular neurocysticercosis. Clin Neurol Neurosurg. 2008, 110:718-721. 10.1016/j.clineuro.2008.03.008

6. Serpa JA, Graviss EA, Kass JS, White AC Jr: Neurocysticercosis in Houston, Texas. Medicine. 2011, 90:81-86. 10.1097/MD.0b013e318206d13e

7. Del Brutto OH, Nash TE, White AC Jr, et al.: Revised diagnostic criteria for neurocysticercosis . J Neurol Sci. 2017, 372:202-210. 10.1016/j.jns.2016.11.045

8. Rajshekhar V: Neurocysticercosis: diagnostic problems and current therapeutic strategies . Indian J Med Res. 2016, 144:319-326. 10.4103/0971-5916.198686 